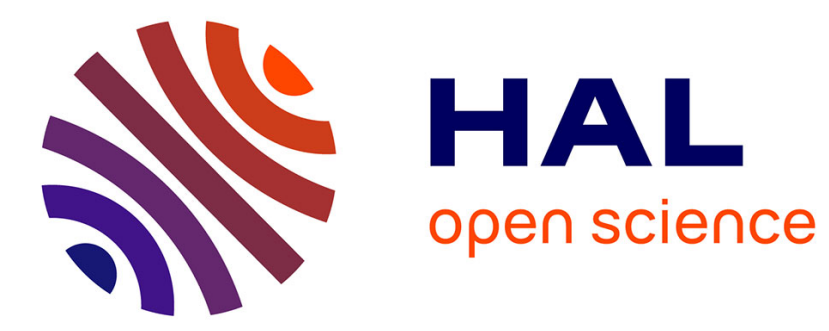

\title{
L'analyse de descriptions verbales dans l'étude des comparaisons de timbres musicaux
}

\author{
V. Nosulenko, E. Samoylenko, S. Mcadams
}

\section{To cite this version:}

V. Nosulenko, E. Samoylenko, S. Mcadams. L'analyse de descriptions verbales dans l'étude des comparaisons de timbres musicaux. Journal de Physique IV Proceedings, 1994, 04 (C5), pp.C5-637-C5-640. 10.1051/jp4:19945137 . jpa-00252814

\section{HAL Id: jpa-00252814 https://hal.science/jpa-00252814}

Submitted on 1 Jan 1994

HAL is a multi-disciplinary open access archive for the deposit and dissemination of scientific research documents, whether they are published or not. The documents may come from teaching and research institutions in France or abroad, or from public or private research centers.
L'archive ouverte pluridisciplinaire HAL, est destinée au dépôt et à la diffusion de documents scientifiques de niveau recherche, publiés ou non, émanant des établissements d'enseignement et de recherche français ou étrangers, des laboratoires publics ou privés. 


\title{
L'analyse de descriptions verbales dans l'étude des comparaisons de timbres musicaux
}

\author{
V.N. NOSULENKO ${ }^{*, * *, * * *}$, E.S. SAMOYLENKO ${ }^{* * *}$ et S. McADAMS ${ }^{*, * * * *}$ \\ * Institut de Psychologie, Académie des Sciences de Russie, Yaroslavskaya Str. 13, 129366 Moscou, Russia \\ ** Laboratoire de Psychologie Expérimentale, CNRS, Université René Descartes, 28 rue Serpente, \\ 75006 Paris, France \\ *** Maison des Sciences de l'Homme, 54 bd Raspail, 75006 Paris, France \\ ${ }^{* * * *}$ IRCAM, 1 place Stravinsky, 75004 Paris, France
}

\begin{abstract}
A study of timbre comparisons was carried out within an experimental paradigm where subjects gave numerical evaluations of the dissimilarity between pairs of complex sounds along with a free verbal description of their similarities and differences. Both professional musicians and nonmusicians participated in the study. A method of verbal protocol analysis was developed in which verbalizations were analyzed from three general points of view: 1) the logical structure of comparison operations, 2) the object-relatedness of the comparison, and 3) the referential content. The protocol analysis method proves to be effective in revealing individual differences and general tendencies in the comparison of complex sounds.
\end{abstract}

\section{INTRODUCTION.}

Le timbre musical présente une diversité de propriétés subjectives dont l'analyse quantitative se révèle parfois difficile. Il s'avère nécessaire donc de l'aborder de plusieurs points de vue expérimentaux. Une étude sur les processus sous-tendant la comparaison des timbres a été menée avec un paradigme expérimental dans lequel les auditeurs avaient deux tâches à accomplir : la première consistait en une évaluation subjective chiffrée du degré de dissemblance entre des paires de sons synthétiques égalisés pour leurs hauteur, sonie et durée, telle qu'elle est pratiquée dans l'analyse multidimensionelle; la seconde consistait en une description verbale libre des similarités et des différences entre les deux timbres de chaque paire. Ensuite les deux types de données ont été analysées et comparées. Notre méthode d'analyse des protocoles verbaux avait été appliquée dans le passé à des comparaisons de stimuli visuels [1] et a été modifiée ensuite pour la modalité sonore [2,3]. Dans la présente étude, nous nous sommes intéressés à la perception auditive dans des groupes d'auditeurs provenant de contextes professionnels différents. L'hypothèse générale de l'étude est que les caractéristiques de la perception auditive et les moyens de description verbale mis en œuvre dépendent du caractère de l'activité professionelle de l'auditeur. En l'occurrence il s'agit d'une comparaison entre musiciens et non-musiciens.

\section{METHODE.}

\subsection{Stimuli.}

Les sons ont été synthétisés sur un Yamaha TX802 FM Tone Generator. Les 17 "instruments" utilisés ont été tirés d'un ensemble de 21 sons synthétiques conçus soit pour imiter certains instruments occidentaux traditionnels (clarinette, clavecin, trompette, etc.), soit pour représenter une sorte de hybride chimérique d'instruments connus (par exemple, le "vibrone" est dérivé du vibraphone et du trombone) [4]. Tous les sons ont été égalisés perceptivement au niveau de leur hauteur (fréquence fondamentale = $311 \mathrm{~Hz}$, ou mi bémol 4), de leur sonie (autour d'une nuance de mezzo forte), et de leur durée 
(approximativement $500 \mathrm{~ms}$ ). Les instruments choisis pour la présente étude ont été utilisés dans cinq comparaisons d' "intervalles" de timbre dans une expérience précédente [5] pour lesquelles les auditeurs musiciens et non musiciens ont donné les résultats les plus nets.

\subsection{Sujets.}

Les sujets, recrutés à Moscou et à Paris, produisaient des protocoles en russe, en français et en anglais. Les 14 sujets russes n'étaient pas des musiciens professionnels tandis que les 9 sujets anglophones et francophones étaient des compositeurs professionnels, dont plusieurs travaillaient dans le domaine de la musique électroacoustique, utilisant les sons synthétiques de façon quotidienne.

\subsection{Procédure.}

Tous les sons utilisés ont été présentés au début de la séance dans un ordre aléatoire afin de familiariser le sujet avec la gamme de variations possible. Ensuite six essais d'entraînement ont été présentés, suivis des 46 essais expérimentaux. Lors de chaque essai, le sujet écoutait une paire de sons. Il pouvait rejouer la paire autant de fois que nécessaire. Il lui était demandé de faire un jugement numérique du dissemblance des deux sons sur une échelle de 1 à 8 , où 1 signifie très similaire et 8 très dissemblable. Il devait ensuite comparer les sons et décrire verbalement leurs similarités et dissemblances en autant de détail que possible. Ces protocoles étaient enregistrés, transcrits textuellement et analysés.

\subsection{Procédure d'analyse des protocoles verbaux.}

Chaque texte verbal produit par le sujet lors de la présentation d'une paire de sons a été analysé selon trois points de vue généraux.

1) La structure logique des opérations de comparaison représentée dans la verbalisation : La première étape consistait à relever les parties des verbalisations qui contenaient des descriptions de similarités ou de dissemblances entre les stimuli. Pour différencier ces deux parties, les constructions syntaxiques et lexicales correspondantes qui existent dans les langues étudiées ont été prises en considération. Ensuite les unités verbales qui caractérisent les similarités ou les dissemblances en termes de paramètres généraux et celles qui les expriment de façon concrète ont été identifiées. Enfin l'analyse distinguait les modes logiques de représentation des différences en termes de comparaisons discrètes et continues : ainsi on classait les unités verbales comme "classificatoires" et "graduelles".

2) La façon de se référer à des sons comparés: A ce niveau on identifiait les unités verbales utilisées pour décrire la nature intégrale d'un son ("Gestalt sonore") et celles faisant référence aux propriétés distinctes du son ("attributs sonores").

3) Le contenu référentiel des unités verbales : Trois catégories principales ont été distinguées : 1) traits verbaux distincts ; 2) représentations verbales intégrées qui contiennent en elles une référence à un objet réel ou à un concept abstrait et qui ne peuvent pas être réduites à une somme des descriptions des traits distincts; 3 ) descriptions des attitudes émotionnelles et évaluatives. Chacune des trois catégories a été subdivisée en catégories plus concrètes selon les tâches et hypothèses de la recherche expérimentale. Par exemple, une représentation verbale intégrée qui contient une référence à un objet a été subdivisée enfin en dispositifs de reproduction sonore et en instruments de musique.

\section{RESULTATS.}

\subsection{Analyse de verbalisations pour paires de timbres donnant des évaluations numériques disparates.}

Pour tester la procédure d'analyse des verbalisations, nous sommes partis du fait qu'il existe des évaluations numériques de dissemblance qui sont parfois très dispersées, certains sujets donnant des évaluations peu élevées (très similaires) et d'autres élevées (très dissemblables) pour une même paire de timbres. Notre hypothèse était que dans ces cas les sujets utilisent des critères divers pour les évaluations et nous supposions que l'analyse verbale dévoilera leur nature. Pour vérifier cette hypothèse nous avons entrepris un triage spécial des données du point de vue des évaluations faites par les sujets : une catégorie "dissemblable" a été constituée pour les jugements supérieurs à 6 , et une catégorie "similaire" pour les jugements inférieurs à 3 .

Voici un exemple des descriptions des différences entre les mêmes timbres faites par le même sujet dans deux séances consécutives :

Séance 1 - (Evaluation $=2)$ "Les deux sons sont très similaires ... parce qu'ils sont tous les deux très artificiels." 
Séance 2 - (Evaluation = 7) "Ce sont ... des instruments différents. En outre ... ils diffèrent par la hauteur."

L'analyse consistait en une comparaison des caractéristiques verbales associées aux grandes et aux petites évaluations de dissemblance. On a comparé les fréquences d'utilisation des différents types d'unités verbales pour la présentation d'une paire de timbres donnée.

L'analyse nous a montré qu'au niveau de la structure logique de la comparaison, les verbalisations de similarités ainsi que de dissemblances entre les sons correspondaient aux évaluations numériques.

1) Les verbalisations des caractéristiques sonores similaires ont été utilisées plus souvent par le groupe de sujets donnant une évaluation peu élevée par rapport au groupe donnant des évaluations élevées pour la même paire de sons. A la deuxième étape de l'analyse (la façon dont les descriptions se réferent à des objets), cette correspondance des fréquences d'utilisation des descriptions de similarité avec les évaluations numériques concerme à la fois les attributs (par exemple "attaque") et les Gestalts sonores (par exemple "un son riche"). Pourtant au niveau du contenu référentiel des verbalisations, une correspondance significative entre les données verbales et psychophysiques a été remarquée seulement dans l'emploi de traits verbaux distincts.

2) Les verbalisations des caractéristiques dissemblables ont été utilisées plus souvent par le groupe de sujets qui donnaient des grandes valeurs de dissemblance que par le groupe donnant des petites valeurs. Cependant, au niveau de la structure logique de la comparaison, il existe une correspondance entre les caractéristiques verbales et les évaluations numériques uniquement pour les unités verbales qui ont un caractère classificatoire, affectant les sons évalués comme dissemblables aux catégories sémantiques différentes ("Le premier a une attaque longue, ... l'autre a une attaque riche."). L'utilisation d'une stratégie de graduation dans les verbalisations de dissemblances ne semble pas être corrélée à la différence perçue entre les sons. Au niveau de la façon dont les descriptions se réfèrent à des objets, la correspondance des caractéristiques verbales aux évaluations numériques concerne à la fois les représentations des attributs et des Gestalts sonores. Néanmoins, comme dans le cas des verbalisations de similarités, au niveau du contenu référentiel, une correspondance significative entre les verbalisations de dissemblance et les évaluations numériques existe seulement pour les traits verbaux distincts : une différence au niveau des traits distincts apparaît plus souvent pour les paires de timbres perçues comme dissemblables.

\subsection{Portraits verbaux des timbres.}

Afin d'évaluer l'effet de l'expérience professionelle, nous avons analysé la fréquence d'utilisation des unités verbales concernant les références aux objets sonores concrets. Nous avons étudié les particularités de la reconnaissance des timbres par les sujets dans les deux catégories : musiciens/nonmusiciens. Notre hypothèse était que les données verbales des musiciens professionnels devaient être plus précises et plus concrètes comparées aux données des sujets non-professionnels.

Pour cette analyse, nous avons calculé le nombre des référents concrets qui caractérisent les instruments de musique. Lors du triage nous avons choisis les unités verbales identifiant clairement un instrument de musique ou une famille d'instruments ("guitare", "cuivre", "vibraphone", "cordes", etc.). Nous avons réunis ces unités verbales dans trois classes générales : "vents", "cordes", "percussions". Une quatrième classe ("autres") correspondait à tous les autres référents. Les unités verbales de cette classe étaient de type plus général ("instrument de musique", par example).

Le résultat de l'analyse témoigne de différences importantes dans la perception des timbres étudiés. Par exemple, le groupe des professionnels identifie la paire de timbres "striano" (hybride entre corde frottée et piano) et trompette plutôt comme des vents, indiquant une perception de similarité de point de vue de leurs références aux instruments de musique. Pour la même paire par contre, le groupe des nonprofessionnels identifie le "striano" comme une corde, tandis que la trompette est toujours identifiée comme un vent. Ceci suggère que les deux sons tout en étant perçus comme similaires par les professionnels, sont perçus comme dissemblables par les non-professionnels.

Les sujets professionnels sont plus précis dans leurs identifications et les non-professionnels utilisent relativement plus souvent les descripteurs faisant partie de la classe générale ("autres"). Ces derniers utilisent un champ de catégories qui est beaucoup moins défini que chez les professionnels. Il faut reconnaître que les sujets professionnels avaient une expérience importante dans l'écoute des sons synthétisés. C'est-à-dire, leurs points de repère auditifs sont étendus vers les sons synthétiques. Il est intéressant tout de même de souligner que même pour les professionels, les timbres ambigus du point de vue de leur identification sont pour la plupart les sons hybridés! 


\section{CONCLUSIONS.}

Les particularités de la perception de différences entre les timbres musicaux se dévoilent à chacun des trois niveaux d'analyse : structure logique des opérations de comparaison représentées dans les verbalisations, référence à des objets dans la comparaison et contenu référentiel de la comparaison. Il existe une différence significative en descriptions de timbres entre les groupes de sujets professionnels et non-professionnels.

Nous pouvons conclure ainsi que notre analyse met en évidence une correspondance entre l'évaluation numérique des "distances" subjectives entre ls sons et la façon dont les sujets décrivent leurs similarités et dissemblances. Ce résultat suggère la possibilité que les descriptions verbales sont organisées de façon adéquates par rapport aux représentations perceptives des sons complexes.

On peut conclure également que la méthode d'analyse des protocoles verbaux proposée est assez efficace pour l'analyse comparative des particularités des verbalisations produites lors des évaluations des dissemblances entre les sons musicaux.

\section{REFERENCES.}

[1] Samoylenko E.S. Comparison operations in cognitive-communication tasks [en russe]. Thèse de doctorat, Institute of Psychology, USSR Academy of Sciences, Moscou (1986).

[2] Nosulenko, V.N. \& Samoylenko, E.S. Perception of sound environment changes determined by technological development of society. 25e Congrès International de Psychologie, Bruxelles, Int. J. Psychol., 27(3/4) (1992) 20-21.

[3] Samoylenko, E.S., McAdams, S. \& Nosulenko, V.N. Méthode d'analyse des verbalisations pour l'étude de la perception des timbres. Programme de collaboration franco-russe : Rapport d'activité février-juillet 1993, Maison des Sciences de l'Homme, Paris, (1993) pp. 9-29.

[4] Wessel, D.L., Bristow, D. \& Settel, Z. Control of phrasing and articulation in synthesis. Proceedings of the 1987 International Computer Music Conference, Computer Music Association, San Francisco, (1987) pp. 108-116.

[5] McAdams, S. \& Cunibile, J.-C. Perception of timbral analogies. Phil. Trans. Roy. Soc. Lond., B 336 (1992) 383-389. 